\title{
CACP syndrome: identification of five novel mutations and of the first case of UPD in the largest European cohort
}

\author{
Sara Ciullini Mannurita ${ }^{1,10}$, Marina Vignoli ${ }^{1,10}$, Lucia Bianchi ${ }^{2}$, Anuela Kondi ${ }^{3}$, Valeria Gerloni ${ }^{4}$, \\ Luciana Breda ${ }^{5}$, Rebecca ten Cate ${ }^{6}$, Maria Alessio $^{7}$, Angelo Ravelli ${ }^{8}$, Fernanda Falcini ${ }^{9}$ and Eleonora \\ Gambineri $^{\star}, 1$
}

Camptodactyly-Arthropathy-Coxa vara-Pericarditis (CACP) syndrome is a rare autosomal recessive disorder caused by mutations in PRG4 gene that encodes for proteoglycan 4, a mucin-like glycoprotein that is the major lubricant for joints and tendon surfaces. The molecular studies reported so far have described the identification of 15 mutations associated with this syndrome and the majority of them were found in families of Arabian origin. Here we report the molecular investigation of the largest European cohort that comprises 13 patients, and allowed the identification of 5 novel mutations and of the first case of CACP syndrome resulting from uniparental disomy of chromosome 1.

European Journal of Human Genetics (2014) 22, 197-201; doi:10.1038/ejhg.2013.123; published online 12 June 2013

Keywords: Camptodactyly-Arthropathy-Coxa vara-Pericarditis syndrome; PRG4 gene; UPD

\section{INTRODUCTION}

Camptodactyly-Arthropathy-Coxa vara-Pericarditis (CACP) syndrome is a rare genetic disorder characterized by autosomal recessive inheritance (MIM 208250).

CACP patients have normal joints at birth, but with aging they experience a noninflammatory hyperplasia of the synoviocytes, which is responsible for the development of camptodactyly and arthropathy that lead to premature joint contracture. ${ }^{1}$ Progressive coxa vara deformity and pericarditis can also occur. ${ }^{2,3}$

The locus associated with this syndrome was identified on human chromosome 1q25-q 31 by homozygosity mapping, ${ }^{1}$ and the PRG4 gene was later identified as the causative gene. ${ }^{4}$ This gene is expressed in several organs, including liver, lung, heart and highly in synovial tissue. Among different transcripts derived from PRG4 gene by alternative splicing, ${ }^{5}$ lubricin is a 1404 amino-acid glycoprotein secreted by fibroblastic synoviocytes, chondrocytes in the superficial zone of articular cartilage and by cell lining of tendon sheaths. It is the major lubricant for joint and tendon surfaces, and is involved in regulating cell growth. ${ }^{6,7}$ A study in $P r g 4^{-1}$ mice has suggested that lubricin is needed to prevent protein deposition onto cartilage from synovial fluid, to inhibit the adhesion of synovial cells to the cartilage surface and to control adhesion-dependent synovial growth. ${ }^{8}$ In addition, the deletion of the gene Prg4 in mice resulted in significant structural and biomechanical changes in the articular cartilage with age, some of which are consistent with osteoarthritic degeneration. ${ }^{9}$ Lubricin contains multiple domains, in particular the large central mucin-like domain encoded by exon 6 , which is rich of repeated motifs for post-translationally O-linked glycosylation. ${ }^{10}$ This region is thought to be the lubricating domain of lubricin, as the sequential removal of the last sugars results in loss of boundary lubricating ability. ${ }^{11}$

To date, 15 mutations in the PRG4 gene have been reported, which are associated with CACP syndrome in 20 affected patients mainly of Arabian origin. ${ }^{4,12-14}$

In the present study, we report the results of PRG4 gene molecular analysis in 13 European patients, from 10 unrelated families, who were diagnosed with CACP syndrome. We identified novel mutations in the cohort analyzed. Furthermore, we describe a case of CACP syndrome, resulting from uniparental isodisomy of chromosome 1.

\section{MATERIALS AND METHODS \\ Patients}

A total of 13 patients from 10 unrelated families were clinically diagnosed with CACP syndrome and analyzed for the presence of sequence mutations in PRG4 gene. Ten patients were Italian, two were Albanian and one was Dutch. Written informed consent was obtained from the patient's parents and the study was approved by the local ethics commitees.

The clinical features of the patients are reported in Table 1. The age-of-onset of symptoms was at birth or in the first years of life. All patients had arthropathy and the majority showed camptodactyly, coxa-vara and synovial hyperplasia, whereas only $30 \%$ of patients exhibited pericarditis.

Genomic DNA was isolated from peripheral blood leukocytes using the QIAamp DNA Blood Mini Kit according to the manifacturer's instructions (Qiagen, Hilden, Germany).

\footnotetext{
${ }^{1}$ Department of 'NEUROFARBA', Section of Child's Health, University of Florence and Anna Meyer Children's Hospital, Florence, Italy; ${ }^{2} U$ nit of Hematology, Azienda OspedalieroUniversitaria Careggi, Florence, Italy; ${ }^{3}$ Pediatrics, Centro Ospedaliero-Universitario 'Madre Teresa', Tirana, Albania; ${ }^{4}$ Department of Rheumatology, Hospital G. Pini, Milan, Italy; ${ }^{5}$ Department of Pediatrics, University of Chieti, Chieti, Italy; ${ }^{6}$ Department of Pediatrics, Leiden University Medical Center, Leiden, The Netherlands; ${ }^{7}$ Department of Pediatrics, University Federico II of Naples, Naples, Italy; ${ }^{8}$ Istituto Giannina Gaslini and University of Genoa, Genoa, Italy; ${ }^{9}$ Department of Internal Medicine, Section of Rheumatology, Transition Clinic, University of Florence, Florence, Italy

*Correspondence: Dr E Gambineri, Department of 'NEUROFARBA', Section of Child's Health, University of Florence and Anna Meyer Children's Hospital, Viale G. Pieraccini 24, Florence 50139, Italy. Tel + 39055 5662405; Fax: + 39055 4221012; E-mail: eleonora.gambineri@unifi.it

10These authors contributed equally to this work.
}

Received 4 March 2013; revised 29 April 2013; accepted 8 May 2013; published online 12 June 2013 
Table 1 Clinical features of all patients

\begin{tabular}{|c|c|c|c|c|c|c|c|c|c|c|c|c|c|}
\hline Clinical manifestations & Pt 1 & Pt 2 & Pt 3 & Pt 4 & Pt 5 & Pt 6 & Pt 7 & Pt 8 & Pt 9 & Pt 10 & Pt 11 & Pt 12 & Pt 13 \\
\hline Gender & Male & Male & Male & Female & Female & Female & Female & Male & Female & Male & Male & Male & Male \\
\hline Age at onset & 3 y & At birth & At birth & $3 y$ & $3 \mathrm{~m}$ & $6 \mathrm{~m}$ & At birth & At birth & At birth & $10 \mathrm{y}$ & $7 \mathrm{~m}$ & At birth & 5 y $6 \mathrm{~m}$ \\
\hline Age at diagnosis & 12 y & $8 \mathrm{y}$ & $5 y$ & $6 y$ & $5 y$ & $10 \mathrm{y}$ & $5 y$ & $3 y$ & at birth & $15 \mathrm{y}$ & $3 y$ & $6 \mathrm{y}$ & 15 y \\
\hline Parents consanguinity & No & No & No & No & No & No & Yes & No & No & No & No & No & No \\
\hline \multicolumn{14}{|l|}{ Camptodactyly } \\
\hline Hands & No & No & No & No & Yes & Yes & Yes & Yes & Yes & No & Yes & Yes & Yes \\
\hline Feet & No & Yes & Yes & No & Yes & Yes & No & No & No & No & No & Yes & Yes \\
\hline \multicolumn{14}{|l|}{ Arthropathy } \\
\hline Elbows & Yes & Yes & Yes & No & No & Yes & Yes & Yes & No & Yes & Yes & Yes & No \\
\hline Wrists & Yes & Yes & No & No & Yes & Yes & Yes & Yes & Yes & Yes & Yes & Yes & Yes \\
\hline Hips & Yes & Yes & Yes & Yes & Yes & Yes & Yes & Yes & No & Yes & No & No & No \\
\hline Knees & Yes & Yes & Yes & Yes & Yes & Yes & Yes & Yes & No & Yes & Yes & Yes & No \\
\hline Ankles & Yes & Yes & Yes & No & Yes & Yes & Yes & Yes & No & Yes & No & No & Yes \\
\hline Pericarditis & Yes & No & No & No & Yes & No & No & Yes & No & Yes & No & No & No \\
\hline \multicolumn{14}{|l|}{ X-ray } \\
\hline Joint erosions & No & No & No & No & Yes & Yes & Yes & No & Yes & Yes & No & No & Yes \\
\hline Coxa vara & Yes & Yes & Yes & No & Yes & Yes & Yes & Yes & No & Yes & Yes & Yes & Yes \\
\hline \multicolumn{14}{|l|}{ Ecography } \\
\hline Joint effusion & No & Yes & Yes & Yes & Yes & Yes & Yes & Yes & No & Yes & Yes & Yes & No \\
\hline Synovial hyperplasia & Yes & Yes & Yes & Yes & Yes & Yes & Yes & Yes & No & Yes & Yes & Yes & Yes \\
\hline \multicolumn{14}{|l|}{ Synovial fluid } \\
\hline Viscous & NA & Yes & Yes & Yes & Yes & Yes & Yes & Yes & No & Yes & Yes & Yes & Yes \\
\hline Honey colored & NA & Yes & Yes & Yes & Yes & Yes & Yes & Yes & No & Yes & Yes & Yes & Yes \\
\hline Low cells count & NA & Yes & Yes & Yes & NA & NA & Yes & Yes & No & No & Yes & Yes & Yes \\
\hline Other affected family members & - & - & - & - & Sister (Pt 6) & Sister (Pt 5) & - & Sister (Pt 9) & Brother (Pt 8) & - & Brother (Pt 12) & Brother (Pt 11) & - \\
\hline
\end{tabular}

Abbreviations: m, months; NA, data not available; Pt, patient; $y$, years..

\section{Sequence analysis}

The entire coding sequence, including exon-intron junctions, was amplified using standard PCR conditions, with the exception of a 800 bp region of exon 6 (c.1092-1871) because of its highly repetitive nature. Primer pairs specific for exons 1, 2, 3, 4 and 7 were taken from elsewhere, ${ }^{4}$ all other primers used in this study can be obtained from the authors. PCR products were sequenced using the BigDye Terminator Cycle Sequencing Kit (Applied Biosystems, Foster City, CA, USA) on an automated ABI PRISM 3130 Genetic Analyzer (Applied Biosystems) and compared with reported reference cDNA sequence (GenBank accession no. NM_005807.3).

\section{Real-time gene-dosage analysis}

Real-time quantitative PCR was performed for PRG4 exons 6 and 12 on a 7500 Fast Real-Time Instrument (Applied Biosystems), using SYBR Green as intercalating fluorescent dye. The GAPDH gene, located on chromosome 12, was used as internal reference locus.

Lymphocyte DNAs from 10 control subjects were used as reference normal samples for the optimization of experimental conditions.

The primer sequences (available upon request) were designed using the Primer 3 software (http://frodo.wi.mit.edu/). ${ }^{15}$ One microliter of sample DNA (20 ng) was added to the PCR reaction mixture containing $2 \times$ SYBR Green Master Mix, and $200 \mathrm{nmol} / \mathrm{l}$ forward and reverse primers in a final volume of $10 \mu$ l. All analyses were performed in triplicate. The PCR amplification profile was as follows: initial denaturation at $95^{\circ} \mathrm{C}$ for $5 \mathrm{~min}$, followed by 40 cycles of denaturation at $95^{\circ} \mathrm{C}$ for $15 \mathrm{~s}$, annealing for $60 \mathrm{~s}$ and extension at $72{ }^{\circ} \mathrm{C}$ for $15 \mathrm{~s}$. Detection of the fluorescent product was carried out during the annealing period. Data analysis was performed using the comparative Delta Delta $\mathrm{Ct}$ $(\Delta \Delta \mathrm{Ct})$ method. ${ }^{16}$ The relative gene copy number was calculated by the expression $2^{-\Delta \Delta \mathrm{Ct}}$.

\section{Microsatellite analysis for UPD}

Ten polymorphic short tandem repeat markers covering both short and long arms of chromosome 1 were analyzed on one patient's genomic DNA and on their parents in order to investigate the parental origin of each copy of chromosome 1. The following microsatellites were explored: D1S199, D1S220 and D1S207 on the short arm, and D1S2344, D1S2635, D1S191, D1S2877, D1S249, D1S2827 and D1S2833 on the long arm. Forward primers were labeled with 6-FAM, TET or HEX fluorescent dyes, and PCR products were analyzed on a automated ABI PRISM 310 Genetic Analyzer (Applied Biosystems). Product size determination was assessed with GeneScan software (Applied Biosystems).

\section{RESULTS}

A total of 13 patients were investigated for the presence of mutations in the PRG4 gene coding sequence. This analysis revealed the presence of six mutations in nine patients (Table 2), five of which were novel mutations (Figure 1). These include a deletion of $5 \mathrm{bp}$ in exon 10 
a Chondroitin Sulphate attachment site
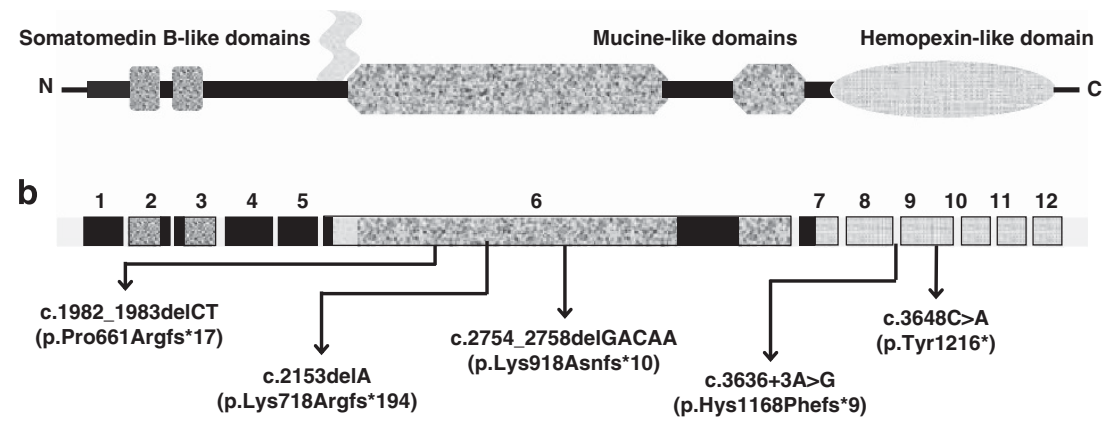

Figure 1 Schematic representation of human lubricin. (a) Protein structure with functional domains; (b) gene structure with location of the novel mutations identified in the present study.

Table 2 PRG4 gene mutations identified

\begin{tabular}{lccl}
\hline Patients & Location & Mutation at cDNA level & $\begin{array}{c}\text { Deduced mutation at } \\
\text { protein level }\end{array}$ \\
\hline 1 & Exon 6 & c.2754_2758delGACAA & p.Lys918Asnfs*10 \\
2 & Intron 8 & c.3636+3A $>$ G & p.Hys1168Phefs*9 \\
3,11 and 12 & Exon 10 & c.3894_3898delGGTTA & p.Val1299Glufs*5 \\
4 & Exon 6 & c.1982_1983delCT & p.Pro661Argfs*17 \\
5 and 6 & Exon 9 & c.3648C $>$ A & p.Tyr1216* \\
10 & Exon 6 & c.2153delA & p.Lys718Argfs*194 \\
\hline
\end{tabular}

aThe identification number of the patients is the one stated in Table 1. *indicate a translation termination codon.

(ref.4) an alteration of the donor splice site region of intron 8, a nonsense mutation in exon 9 and three small deletions located in the inner region of exon 6 .

The potential pathogenicity of the splice site mutation has been examined using different programs, including the splice site prediction tool from the Berkeley Drosophila Genome Project web site (http://www.fruitfly.org/seq_tools/splice.html), ${ }^{17}$ NetGene2, available from the Center of Biological Sequence analysis (http://www.cbs. dtu.dk/services/NetGene2/) ${ }^{18,19}$ and Alamut Software version 2.2e (Interactive Biosoftware, Rouen, France). All programs showed a marked decrease of the score or the absence of the donor splice site with an eligible value. The alteration of the donor splice site could lead to the skipping of exon 8 , finally resulting in a frameshift of the protein sequence and in the formation of a stop codon after nine amino-acid residues. The nonsense mutation and the small deletions are also predicted to create a stop codon. This could result either in the expression of a truncated and non functional protein or in the absence of lubricin synthesis. The latter possibility has been previously proved for some nonsense/frameshift PRG4 mutations as a consequence of nonsense-mediated mRNA decay. ${ }^{20}$ Unfortunately, due to the unavailability of synovial fluid from the patients, the structure of the protein could not be investigated.

All of the mutations were present in a homozygous state in the patients, whereas none of them were observed in 200 control chromosomes and in 6500 exomes of the Exome Sequencing Project (Exome Variant Server, NHLBI GO Exome Sequencing Project (ESP), Seattle, WA, USA; URL: http://evs.gs.washington.edu/EVS/).

In the case of patient 4 , amplification and sequencing of PRG4 exon 6 in the parents showed that the father was a heterozygous carrier of the c.1982-1983delCT mutation, but the mother did not have this alteration (Figure 2). This could be either due to the presence of a large deletion encompassing the portion of exon 6 investigated that is

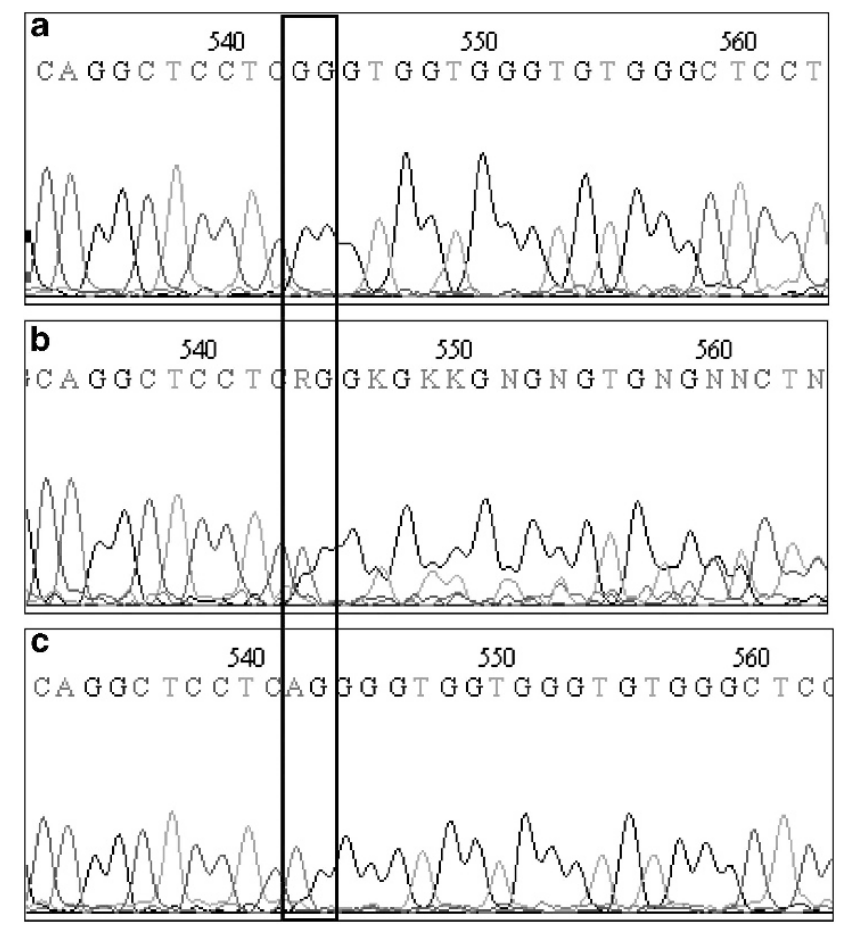

Figure 2 Electropherogram showing the reverse strand sequence of exon 6 of the family of patient 4. (a) Proband sequence harboring the homozygous deletion c.1982_1983delCT; (b) father sequence harboring the deletion in heterozygosity; (c) mother sequence, wild type.

not detectable by conventional PCR-based methods or due to the occurrence of uniparental disomy in which the proband inherits both copies of a chromosome from only one parent.

Gene-dosage analysis performed by real-time quantitative PCR was able to determine the allelic copy number and demonstrated the absence of large deletions within chromosome 1 of the proband and the mother.

The subsequent analysis of microsatellite markers spanning the whole chromosome 1 revealed that the patient is homozygous for each short tandem repeat tested, and seven informative loci indicated that both copies of the chromosome were inherited from the father, thus showing the existence of a paternal uniparental isodisomy (Figure 3).

\section{DISCUSSION}

PRG4 mutations has been described in 20 CACP cases of different ethnicities but mainly among patients of Arabian origin. In the 


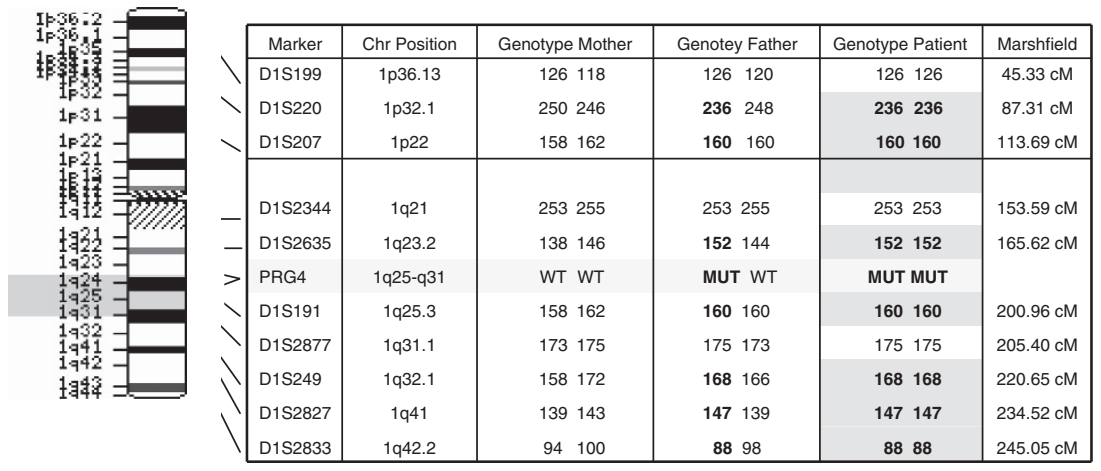

Figure 3 Schematic representation of the paternal UPD1. The different markers used for segregation analysis are shown on the chromosome. The table gives the position for each marker according to the banding pattern and Marshfield (cM) map, and the genotypes for both parents and the index patient. WT, wild-type allele; MUT, mutated allele.

present study, we report the results of the molecular investigation of PRG4 gene in the largest European cohort, which includes 13 CACP patients belonging to 10 unrelated families.

The clinical features exhibited by our series of patients reflect the classical signs of the syndrome and are consistent with those reported in previous studies, such as arthropathy of large joints in all patients described, camptodactyly and coxa vara in the majority of them, and pericarditis reported in only $20 \%$ of cases. ${ }^{1,21}$ In particular, our case series includes patients that exhibit all major clinical signs (Pt 5 of Table 1) and patients presenting only arthropathy, without camptodactyly pericarditis and coxa vara (Pt 4 of Table 1), confirming the clinical variability reported so far within and across families. We have identified six different sequence variants in nine subjects. One of them has been reported earlier as a heterozygous alteration, ${ }^{4}$ whereas five were novel mutations.

Interestingly, three of the new mutations detected were located in exon 6 , which accounts for $67 \%$ of the entire coding sequence and encodes for the large mucin-like domain. These new mutations increase the number of variations identified in this portion of the gene, overall 14 out of 20 mutations in PRG4 occur in exon 6. This finding strengthens the results of in vitro studies, suggesting that exon 6 encodes the major lubricating domain of lubricin.

Like the majority of the variations already reported, all mutations identified in our patients are nonsense mutations, frameshift deletions or splicing defects that are predicted to lead to the creation of a premature termination codon and subsequently to the lack of lubricin synthesis, suggesting that CACP syndrome is mainly due to the complete absence of the proteoglycan 4 protein.

Therefore, this study shows that although the alterations identified are different in terms of type of mutation and localization within the gene, they share the same predicted effect on protein synthesis, thus underlying the genetic homogeneity of the syndrome, which is in contrast with the clinical variability. This observation, together with the analysis of the different clinical manifestations of our patients and in particular of two sisters harboring the same mutation ( $\mathrm{Pt} 5$ and Pt 6 of Table 1), do not allow us to describe the presence of a phenotype-genotype correlation.

Interestingly, we found a patient with a homozygous mutation in $P R G 4$ gene in which additional investigations revealed complete paternal isodisomy of chromosome 1 . This is the first report of CACP syndrome caused by uniparental inheritance. It is noteworthy that in this case, we did not expect a different phenotype, as it might be predicted when UPD affect other chromosomes, and this is due to the absence of imprinting effects on chromosome 1 as reported in other studies. $^{22}$ The determination of the occurrence of UPD is important for the parents in regard to the estimation of the recurrence risk which, in this case, is below the $25 \%$ risk for an autosomal recessive inheritance.

In conclusion, this report brings new insights into CACP syndrome. First of all, it represents the molecular investigation of the largest European cohort of CACP patients and shows the presence of several distinct novel mutations of PRG4 gene. In particular, it revealed the absence of a single or few recurrent mutations as responsible of the phenotype, differently from other monogenic diseases where often a single mutation is causative of the majority of cases. ${ }^{23}$ Our results strengthen previous data obtained in CACP patients from other countries, and emphasize the existence of private pathogenic mutations located in different exons. Thus, the analysis of the entire coding sequence of $P R G 4$ gene is essential for the molecular diagnosis of CACP syndrome, with a particular attention to the sequence investigation of exon 6 in which the majority of mutations occur. However, although PRG4 gene has to be consider the major gene linked to the onset of CACP syndrome, a clear genotypephenotype correlation has not been shown so far. Further molecular studies on large cohort of CACP patients could be useful to clarify the clinical variability of the syndrome.

Our results indicate also that, although our patient represents a unique example of CACP syndrome caused by UPD, it should be regarded as a mode of inheritance in cases of CACP when the patient appears homozygous for a mutation found in only one parent.

\section{CONFLICT OF INTEREST}

The authors declare no conflict of interest.

\section{ACKNOWLEDGEMENTS}

We are grateful to all the patients and their parents for their kind cooperation. This work was supported by the Italian Ministry for University (MIUR; ex-60\%) to EG.

1 Bahabri SA, Suwairi WM, Laxer RM, Polinkovsky A, Dalaan AA, Warman ML: The camptodactyly-arthropathy-coxa vara-pericarditis syndrome: clinical features and genetic mapping to human chromosome 1. Arthritis Rheum 1998; 41: 730-735.

2 Martinez-Lavin M, Buendia A, Delgado $\mathrm{E}$ et al: A familial syndrome of pericarditis, arthritis, and camptodactyly. New Eng/ J Med 1983; 309: 224-225.

3 Bulutlar G, Yazici H, Ozdogan H, Schreuder I: A familial syndrome of pericarditis, arthritis, camptodactyly, and coxa vara. Arthritis Rheum 1986; 29: 436-438. 
4 Marcelino J, Carpten JD, Suwairi WM et al: CACP, encoding a secreted proteoglycan, is mutated in camptodactyly-arthropathy-coxa vara-pericarditis syndrome. Nat Genet 1999; 23: 319-322.

5 Ikegawa S, Sano M, Koshizuka Y, Nakamura Y: Isolation, characterization and mapping of the mouse and human PRG4 (proteoglycan 4) genes. Cytogenet Cell Genet 2000; 90: 291-297.

6 Jay GD, Britt DE, Cha CJ: Lubricin is a product of megakaryocyte stimulating factor gene expression by human synovial fibroblasts. J Rheumatol 2000; 27: 594-600.

7 Rees SG, Davies JR, Tudor D et al: Immunolocalisation and expression of proteoglycan 4 (cartilage superficial zone proteoglycan) in tendon. Matrix Biol 2002; 21: 593-602.

8 Rhee DK, Marcelino J, Baker M et al: The secreted glycoprotein lubricin protects cartilage surfaces and inhibits synovial cell overgrowth. J Clin Invest 2005; 115: 622-631.

9 Coles JM, Zhang L, Blum JJ et al: Loss of cartilage structure, stiffness, and frictional properties in mice lacking PRG4. Arthritis Rheum 2010; 62: 1666-1674.

10 Jay GD, Harris DA, Cha CJ: Boundary lubrication by lubricin is mediated by O-linked beta(1-3)Gal-GalNAc oligosaccharides. Glycoconj J 2001; 18: 807-815.

11 Jay GD: Characterization of a bovine synovial fluid lubricating factor. I. Chemical, surface activity and lubricating properties. Connect Tissue Res 1992; 28: 71-88.

12 Alazami AM, Al-Mayouf SM, Wyngaard CA, Meyer B: Novel PRG4 mutations underlie CACP in Saudi families. Hum Mutat 2006; 27: 213.

13 Basit S, Iqbal Z, Umicevic-Mirkov M et al: A novel deletion mutation in proteoglycan-4 underlies camptodactyly-arthropathy-coxa-vara-pericarditis syndrome in a consanguineous pakistani family. Arch Med Res 2011; 42: 110-114.

14 Akawi NA, Ali BR, Al-Gazali L: A novel mutation in PRG4 gene underlying camptodactyly-arthropathy-coxa vara-pericarditis syndrome with the possible expansion of the phenotype to include congenital cataract. Birth Defects Res A Clin Mol Teratol 2012; 94: 553-556.

15 Rozen S, Skaletsky H: Primer3 on the WWW for general users and for biologist programmers. Methods Mol Biol 2000; 132: 365-386.

16 Livak KJ, Schmittgen TD: Analysis of relative gene expression data using real-time quantitative PCR and the 2(-Delta Delta C(T)) Method. Methods 2001; 25: 402-408.

17 Reese MG, Eeckman FH, Kulp D, Haussler D: Improved splice site detection in Genie. J Comp Biol 1997; 4: 311-323.

18 Hebsgaard SM, Korning PG, Tolstrup N, Engelbrecht J, Rouze P, Brunak S: Splice site prediction in Arabidopsis thaliana DNA by combining local and global sequence information. Nucleic Acids Res 1996; 24: 3439-3452.

19 Brunak S, Engelbrecht J, Knudsen S: Prediction of human mRNA donor and acceptor sites from the DNA sequence. J Mol Biol 1991; 220: 49-65.

20 Rhee DK, Marcelino J, Al-Mayouf S et al: Consequences of disease-causing mutations on lubricin protein synthesis, secretion, and post-translational processing. J Biol Chem 2005; 280: 31325-31332.

21 Albuhairan I, Al-Mayouf SM: Camptodactyly-arthropathy-coxavara-pericarditis syndrome in Saudi Arabia: Clinical and molecular genetic findings in 22 patients. Semin Arthritis Rheum 2013 (in press).

22 Nimmo G, Monsonego S, Descartes M, Franklin J, Steinberg S, Braverman N: Rhizomelic chrondrodysplasia punctata type 2 resulting from paternal isodisomy of chromosome 1. Am J Med Genet A 2010; 152A: 1812-1817.

23 Richette P, Bardin T, Stheneur C: Achondroplasia: from genotype to phenotype. Joint Bone Spine 2008; 75: 125-130. 